\section{KCC2 : nouvelle cible thérapeutique pour le traitement de pathologies neurologiques}

Marc J. Bergeron, Annie Castonguay, Yves De Koninck
> La famille SLC12 (solute carrier, transporteurs de solutés) des cotransporteurs cation- $\mathrm{Cl}^{-}$représente les produits de neuf gènes dont sept qui codent pour des protéines dont la fonction et les rôles physiologiques sont bien caractérisés. Parmi ces molécules, les cotransporteurs $\mathrm{Na}^{+}-\mathrm{K}^{+}-\mathrm{Cl}^{-}$isoforme l ( $\mathrm{NKCCl}$ ), et plus particulièrement $\mathrm{K}^{+}-\mathrm{Cl}^{-}$isoforme 2 (KCC2), jouent un rôle clé dans la régulation de l'activité des neurones du système nerveux central (SNC) en modulant principalement la concentration de $\mathrm{Cl}^{-}$intracellulaire $\left(\left[\mathrm{Cl}^{-}\right]_{\mathrm{i}}\right)$.

Dans les neurones immatures, la $\left[\mathrm{Cl}^{-}\right]_{\mathrm{i}}$ est élevée conséquemment à une forte expression de $\mathrm{NKCCl}$ qui couple le mouvement des ions $\mathrm{Na}^{+}$et $\mathrm{K}^{+}$à celui du $\mathrm{Cl}^{-}$vers l'intérieur des neurones. Cela crée un effet dépolarisant voire, à la limite, excitateur en réponse au neurotransmetteur acide $\gamma$-aminobutyrique (GABA) qui laisse passer le $\mathrm{Cl}^{-}$par ses canaux de type $A$ ouverts (Figure I). En revanche, dans les neurones matures, la $\left[\mathrm{Cl}^{-}\right]_{i}$ est maintenue basse par une forte expression de KCC2 qui couple le mouvement des ions $\mathrm{K}^{+}$à celui $\mathrm{du} \mathrm{Cl}^{-}$vers l'extérieur des neurones, induisant un effet hyperpolarisant et fortement inhibiteur du GABA (Figures 1 et 2A). Ainsi, KCC2 joue un rôle physiologique très important chez l'adulte dans le maintien de l'inhibition post-synaptique, qui prévient une surexcitabilité neuronale [1].

Un dérèglement de l'activité (et/ou de l'expression) de KCC2 entraînera donc un dysfonctionnement du contrôle de l'activité neuronale. D'ailleurs, il semble que la perte d'activité (et/ou d'expression) de KCC2 sous-tende plusieurs désordres neurologiques et psychiatriques tels que l'épilepsie, le stress, l'anxiété, la spasticité motrice, l'hyperalgésie paradoxale induite par la morphine, la douleur chronique, la schizophrénie, voire même l'autisme [2].

\section{Expression de KCC2 et blessure} nerveuse périphérique

Dans un modèle animal de douleur chronique (constriction du nerf scia-
Centre de recherche de l'Institut universitaire en santé mentale de Québec, 2601, de la Canardière, G1) 2 G3 Québec, Canada. yves.dekoninck@neuro.ulaval.ca marc.bergeron.2@ulaval.ca tique de rat avec un tube constricteur) (Figure 2B), nous avons démontré que la blessure nerveuse périphérique induisait une baisse d'expression de KCC2 et une perturbation de l'homéostasie du $\mathrm{Cl}^{-}$ dans les neurones de la corne dorsale superficielle de la moelle épinière [3]. Nous avons par la suite montré que cette baisse d'expression de KCC2 dans la corne dorsale superficielle de la moelle épinière était le résultat de la libération

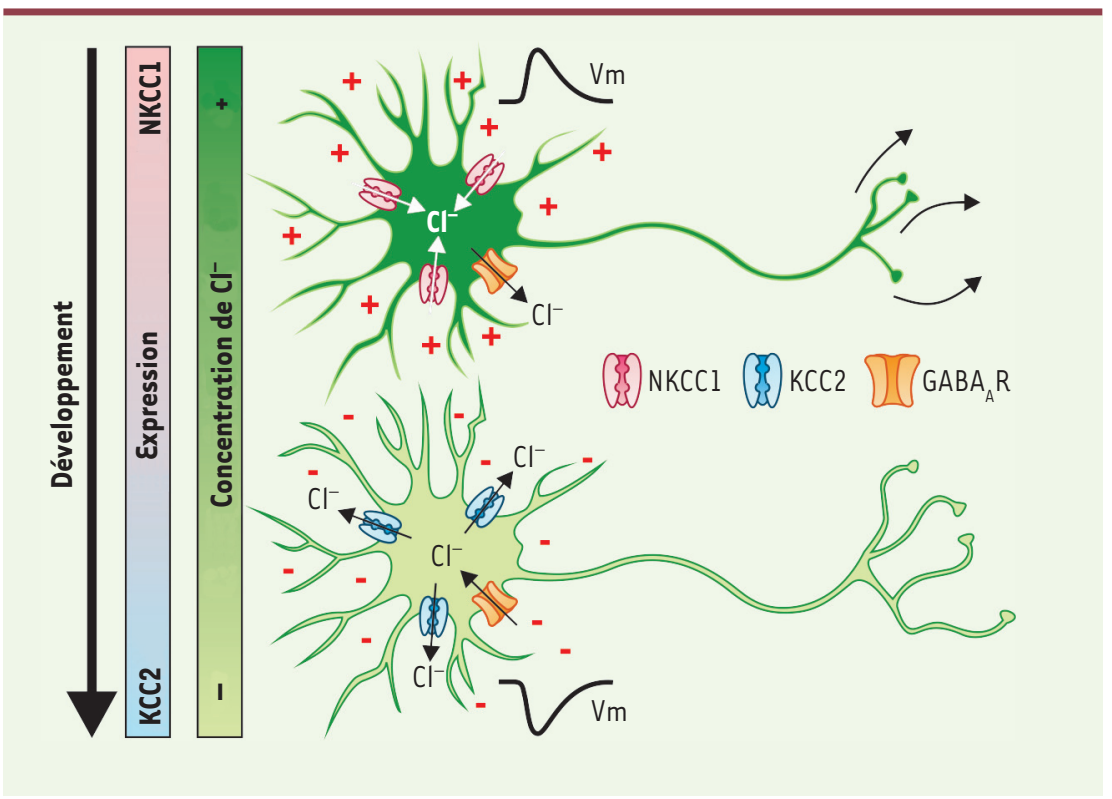

Figure 1. Effet du GABA sur les neurones immatures et matures. Au stade embryonnaire (en haut), l'activation des récepteurs $G A B A_{A}$ et de la glycine $\left(G A B A_{A} / G l y\right)$ engendre une dépolarisation (+) dûe à une forte expression de $\mathrm{NKCCl}$ (rouge) qui importe le $\mathrm{Cl}^{-}$à l'intérieur de la cellule, et une expression quasi-nulle de $\mathrm{KCC}$ (bleu), qui exporte le $\mathrm{Cl}^{-}$hors de la cellule. Cette forte $\left[\mathrm{Cl}^{-}\right]_{\mathrm{i}}$ (vert) entraîne une dépolarisation du potentiel membranaire $\left(\mathrm{V}_{\mathrm{m}}\right)$ lors de l'ouverture des canaux $\mathrm{Cl}^{-}$. Cela confère un rôle trophique au GABA et stimule l'interconnectivité neuronale (flèches). En revanche, à l'âge adulte (en bas), l'activation des récepteurs $G_{A B A} / G l y$ induit une hyperpolarisation (-) dûe à une forte expression de KCC2 et une expression quasi-nulle de NKCCl. Ceci entraîne une baisse de la $\left[\mathrm{Cl}^{-}\right]_{i}$ et une hyperpolarisation du $\mathrm{V}_{\mathrm{m}}$ lors de la stimulation des récepteurs $G_{A B A_{A}} / G l y$; d'où leur rôle principalement inhibiteur. 


\section{(ई夕) KCC2 \\ $\int G_{A B A} R$ \\ $\int$ TrkB \\ Yh Microglie}

Tube Tube Microglie constricteur activée

A

B

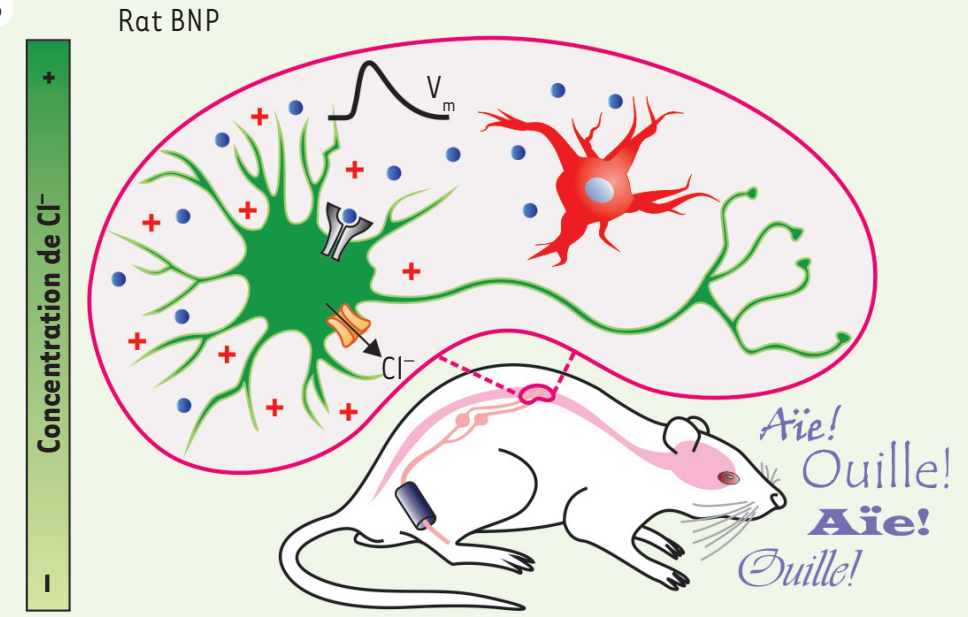

C

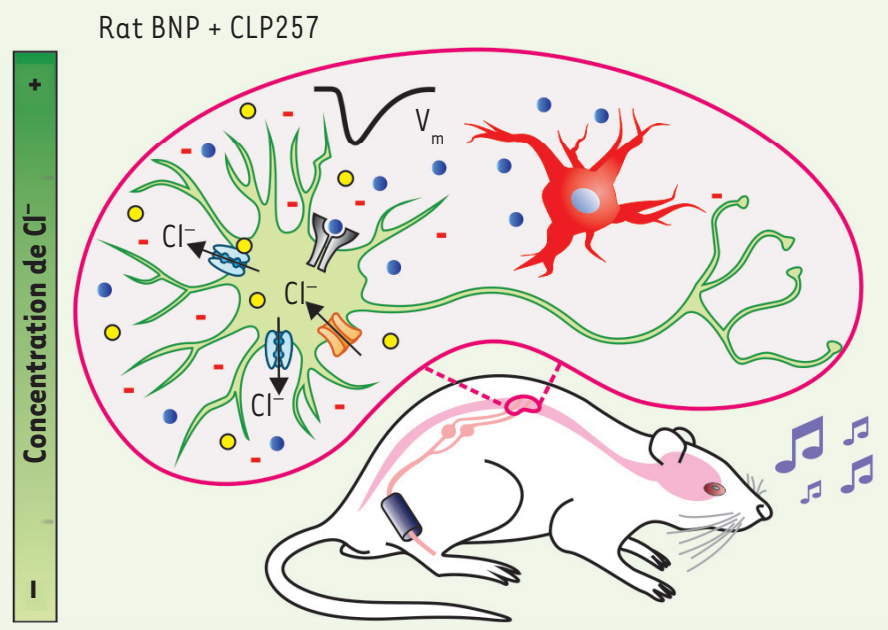

Figure 2. Effet du CLP257 dans un modèle animal de douleur chronique. A. En condition normale, la microglie est inactive et le neurone possède des propriétés synaptiques inhibitrices (-), une concentration intracellulaire basse en $\mathrm{Cl}^{-}\left(\left[\mathrm{Cl}^{-}\right]_{\mathrm{i}}\right.$; vert $)$ et un potentiel membranaire $\left(\mathrm{V}_{\mathrm{m}}\right)$ hyperpolarisé lors de l'ouverture des canaux $\mathrm{Cl}^{-}$. B. Chez un rat avec une blessure nerveuse périphérique (BNP), la constriction du nerf sciatique induit une activation de la microglie qui sécrète du BDNF. La liaison du BDNF au récepteur TrkB induit une baisse d'expression membranaire de KCC2 et conséquemment une hausse de la $\left[\mathrm{Cl}^{-}\right]_{\mathrm{i}}$. Cette hausse cause la dépolarisation du potentiel d'équilibre $\mathrm{du}^{\mathrm{Cl}^{-}}$; il s'ensuit une hyperexcitabilité neuronale $(+)$ responsable d'une hyperalgésie. $C$. Chez un rat ayant une blessure, la molécule CLP257 contrecarre le déficit d'extrusion de $\mathrm{Cl}^{-}$via une augmentation de l'expression membranaire de KCC2, qui entraîne une baisse de la $\left[\mathrm{Cl}^{-}\right]_{\mathrm{i}}$, et un retour à une excitabilité normale.

du facteur neurotrophique brain-derived neurotrophic factor (BDNF) par la microglie activée (Figure 2B) [4]. Nous savons aussi que le BDNF produit ses effets en se liant spécifiquement aux récepteurs à activité tyrosine kinase de type B (TrkB). Cette liaison déclenche l'activation de différentes voies de signalisation intracellulaires qui agissent en modulant l'expression/activité de KCC2 principalement au niveau transcriptionnel et de la synthèse protéique (Figure 3A) [5]. Lors de douleurs neuropathiques chroniques, le BDNF joue donc un rôle prépondérant dans la régulation de KCC2 et, par conséquent, sur l'efficacité de l'inhibition neuronale via la modulation du gradient transmembranaire de $\mathrm{Cl}^{-}$.

\section{Activer KCC2 pour restaurer le potentiel d'inhibition neuronale} Dans ce contexte, il était intéressant de développer des activateurs du KCC2, dans le but de restaurer les propriétés inhibitrices de la transmission GABA/ glycine liées à un défaut de l'homéostasie $\mathrm{du} \mathrm{Cl}^{-}$. C'est ce que notre équipe a 


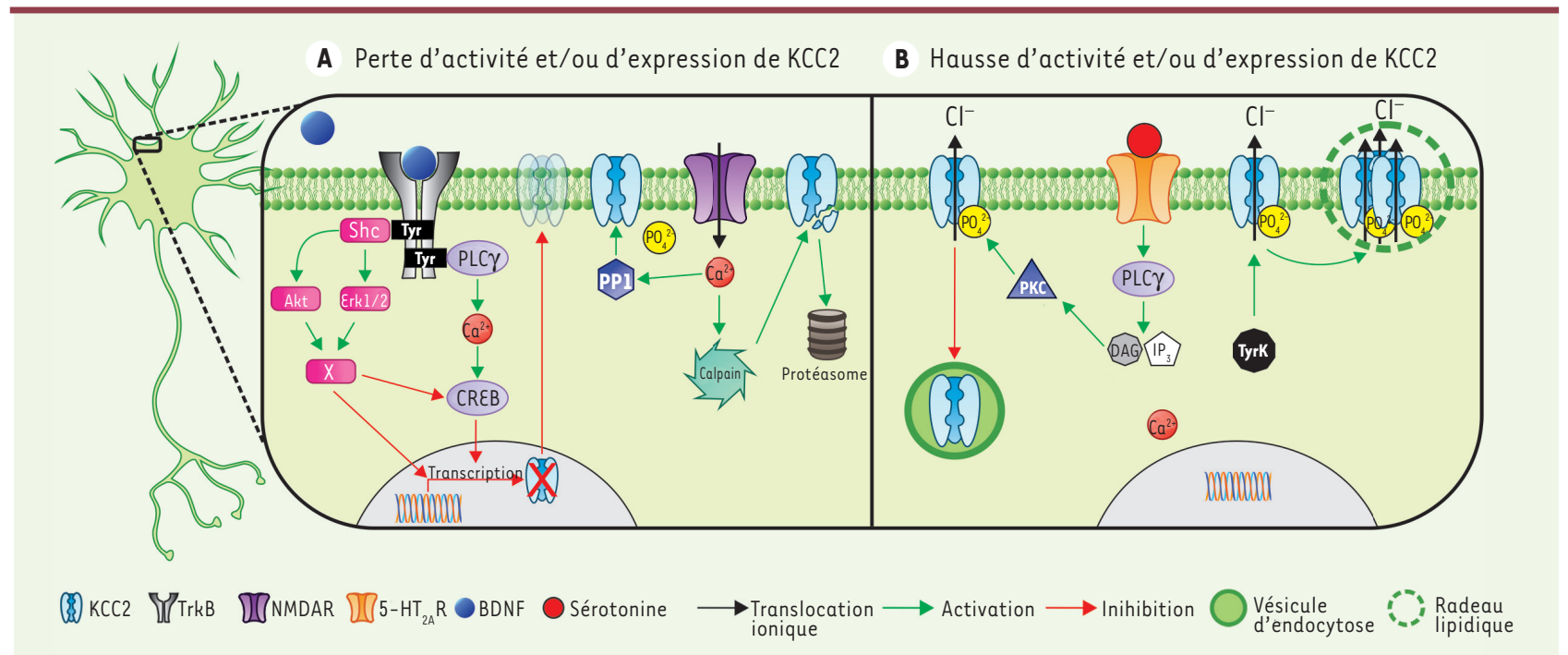

Figure 3. Mécanismes moléculaires impliqués dans la régulation de KCC2. A. Voies de signalisation diminuant l'activité et/ou l'expression de KCC2. La signalisation TrkB/BDNF modulerait l'expression de KCC2 à la baisse en recrutant deux cascades intracellulaires : (1) la voie de la phospholipase $C \gamma(P L C \gamma)$ et (2) la voie de la Src homology 2 domain containing transforming protein (Shc). La voie de la PLC $\gamma$ modulerait la biosynthèse (transcription génique et traduction protéique) en stimulant en aval le facteur de transcription CAMP responses element-binding protein (CREB), alors que la voie de la Shc le ferait en stimulant les voies des protéines kinases Akt et Erk 1/2. Ces dernières activeraient en aval une (ou des) protéine(s) « $X$ » qui modulerai(en)t la biosynthèse directement ou via le facteur CREB. L'entrée de Ca ${ }^{2+}$ via les récepteurs NMDA cause une baisse de l'activité de transport de KCC2 due à sa déphosphorylation par la Ser/Thr protéine phosphatase 1 (PPl), et une perte de son activité/expression due à sa protéolyse par une calpaïne. $B$. Voies de signalisation augmentant l'activité et/ou l'expression de $\mathrm{KCC2}$. La phosphorylation ( $\mathrm{PO}_{4}{ }^{2-}$ ) de $\mathrm{KCC} \mathrm{par}$ une $\mathrm{PKC}$ activée via les récepteurs $5-\mathrm{HT}$ de type $2 \mathrm{~A}\left(5-\mathrm{HT}_{2 \mathrm{~A}}\right)$ de la sérotonine, par exemple, stabilise son expression fonctionnelle à la membrane plasmique par l'inhibition de son endocytose. La phosphorylation $\left(\mathrm{PO}_{4}{ }^{2-}\right)$ de $\mathrm{KCC} 2$ par une protéine tyrosine kinase (TyrK) induit son oligomérisation et sa translocation dans des radeaux lipidiques pour augmenter sa fonction.

cherché à faire. Dans un récent travail [2], nous avons développé un test nous permettant de déterminer l'efficacité d'activateurs de KCC2 à l'aide de lignées cellulaires exprimant de manière stable le senseur ratiométrique de $\mathrm{Cl}^{-}$Cloméléon ${ }^{1}$ [6]. Plus de 95000 composés ont été testés pour leur capacité à restaurer l'extrusion de $\mathrm{Cl}^{-}$. Parmi ceux-ci, 78 ont été sélectionnés, puis de nouveau testés, pour leur sélectivité envers KCC2 et leur faible toxicité, menant à l'identification d'un composé prometteur: le $\mathrm{CL}-058$. Plus de 300 analogues de $\mathrm{CL}-058$ ont alors été synthétisés afin d'amé-

\footnotetext{
${ }^{1}$ Clomeleon est une protéine de fusion associant deux fluorophores: la protéine yellow fluorescent protein (YFP) sensible au chlore, et la protéine cyan fluorescent protein (CFP), insensible au $\mathrm{Cl}^{-}$. La proximité physique des deux fluorophores entraîne un transfert d'énergie (fluorescence resonance energy transfer [FRET]). La fluorescence YFP étant abolie (quenching) par le $\mathrm{Cl}^{-}$, l'émission calculée par le rapport FRET (YFP/CFP) est inversement proportionnelle à la concentration de $\mathrm{Cl}$ -
}

liorer son efficacité envers KCC2, ses caractéristiques pharmacologiques et sa biodisponibilité. La molécule CLP257 a été retenue, principalement grâce à sa meilleure stabilité chimique et ses propriétés générales compatibles avec l'élaboration de médicaments (voir [2]).

Dans ce travail, nous avons d'abord caractérisé CLP257 in vitro du point de vue fonctionnel et moléculaire sur des tranches de corne dorsale superficielle de la moelle épinière de rats naïfs, traitées ou non avec du BDNF, et de rats chez lesquels une blessure nerveuse périphérique a été faite (Figures $2 A-C$ ). Dans ces tranches, nous avons montré, par électrophysiologie et imagerie (durée de vie de fluorescence de l'indicateur de $\mathrm{Cl}^{-} M(A \varepsilon)$, que le CLP257 améliore l'efficacité d'extrusion du $\mathrm{Cl}^{-}$par $\mathrm{KCC} 2$ et restaure le potentiel d'inhibition neuronal du $\mathrm{Cl}^{-}$à des niveaux comparables à ceux qui sont observés chez des animaux naïfs non traités. De plus, des manipulations biochimiques (par exemple la biotinylation de surface cellulaire et l'immunobuvardage de type Western) ont permis de montrer que le CLP257 exerce ses effets en stimulant l'expression de KCC2 à la membrane plasmique (Figure 2C) sans changer l'expression totale de cette protéine, suggérant l'implication de mécanismes post-traductionnels plus que transcriptionnels et traductionnels (biosynthèse).

Nous avons par la suite évalué in vivo l'efficacité et la pharmacocinétique de CLP257, administré à des rats naïfs et soumis à une blessure nerveuse périphérique. Les réponses électrophysiologiques évoquées par la stimulation mécanique de la patte et enregistrées au niveau de la corne dorsale superficielle de la moelle épinière d'animaux naïfs et soumis à une blessure nerveuse 
périphérique (Figure $2 A-C$ ) ont montré que le CLP257 restaure l'inhibition des circuits impliqués [2]. Toutefois, la très faible biodisponibilité du CLP257 ne permettait pas d'obtenir des effets analgésiques suffisants et prolongés. Nous avons contourné ce problème en synthétisant une prodrogue, le CLP290. Celle-ci libère graduellement le métabolite actif CLP257 et permet d'améliorer significativement la pharmacocinétique. L'administration orale de CLP290 a un effet analgésique comparable à celui de la prégabaline (un analgésique très employé pour soigner les douleurs neuropathiques). À des doses analgésiques équipotentielles, le CLP290 provoque cependant moins d'effets secondaires moteurs que la prégabaline [2]. Ces travaux in vivo ont donc permis, d'une part, de corroborer nos résultats obtenus in vitro et, d'autre part, de démontrer clairement le potentiel analgésique des molécules CLP257 et CLP290.

\section{Conclusion et perspectives}

Les mécanismes moléculaires qui soustendent l'effet du CLP257 sur KCC2 demeurent toutefois méconnus. Plusieurs mécanismes de régulation connus pour baisser ou augmenter l'activité et/ ou l'expression de KCC2 méritent d'être explorés. La Figure 3 résume certains mécanismes de signalisation endogènes qui ont été récemment mis en lumière $[5,7-10]$. Il reste néanmoins beaucoup de travail à faire pour bien cerner toute la complexité des mécanismes impliqués dans la régulation du KCC2, notamment en conditions normales et pathologiques.

L'aspect le plus prometteur de nos travaux récents présentés ci-dessus, et de ceux d'autres groupes [7], est la démonstration qu'il est possible de corriger un déficit d'activité du KCC2 et non seulement d'en prévenir la perte d'activité. Il est donc possible d'envisager des traitements curatifs et non plus simplement préventifs. En outre, l'absence d'effets secondaires néfastes lorsque l'on augmente l'activité du KCC2 montre qu'il s'agit d'une cible adaptée au développement de nouveaux médicaments destinés au traitement de maladies neurologiques et mentales. La caractérisation plus approfondie des mécanismes moléculaires qui sous-tendent la régulation du KCC2 constitue donc une étape importante vers l'élaboration de cette nouvelle génération de médicaments. $\diamond$ KCC2: a new therapeutical target for the treatment of neurological diseases

\section{LIENS D'INTÉRÊT}

Les auteurs déclarent n'avoir aucun lien d'intérêt concernant les données publiées dans cet article.

\section{RÉFÉRENCES}

1. Doyon N, Prescott SA, Castonguay A, et al. Efficacy of synaptic inhibition depends on multiple, dynamically interacting mechanisms implicated in chloride homeostasis. PLoS Comput Biol 2011 ; 7 : e1002149.

2. Gagnon M, Bergeron MJ, Lavertu G, et al. Chloride extrusion enhancers as novel therapeutics for neurological diseases. Nat Med 2013 ; 19 : 1524-8.

3. Coull JA, Boudreau D, Bachand K, et al. Trans-synaptic shift in anion gradient in spinal lamina I neurons as a mechanism of neuropathic pain. Nature $2003 ; 424$ : 938-42.

4. Coull JA, Beggs S, Boudreau D, et al. BDNF from microglia causes the shift in neuronal anion gradient underlying neuropathic pain. Nature 2005 ; 438 : 1017-21.

5. Rivera C, Voipio J, Thomas-Crusells J, et al. Mechanism of activity-dependent downregulation of the neuronspecific K-Cl cotransporter KCC2. J Neurosci 2004 ; 24 : 4683-91.

6. Kuner T, Augustine GJ. A genetically encoded ratiometric indicator for chloride: capturing chloride transients in cultured hippocampal neurons. Neuron $2000 ; 27: 447-59$.

7. Bos R, Sadlaoud K, Boulenguez P, et al. Activation of 5-HT2A receptors upregulates the function of the neuronal K-Cl cotransporter KCC2. Proc Natl Acad Sci USA 2013 ; 110 : 348-53.

8. Lee $\mathrm{HH}$, Walker JA, Williams JR, et al. Direct protein kinase $\mathrm{C}$-dependent phosphorylation regulates the cell surface stability and activity of the potassium chloride cotransporter KCC2. J Biol Chem 2007 ; 282 : 29777-84.

9. Lee HH, Deeb TZ, Walker JA, et al. NMDA receptor activity downregulates $\mathrm{KCC} 2$ resulting in depolarizing GABAA receptor-mediated currents. Nat Neurosci $2011 ; 14: 736-43$.

10. Watanabe M, Wake H, Moorhouse AJ, et al. Clustering of neuronal $\mathrm{K}+-\mathrm{Cl}$ - cotransporters in lipid rafts by tyrosine phosphorylation. J Biol Chem 2009 ; 284 : 27980-8.

\begin{tabular}{|c|c|}
\hline $\begin{array}{l}\text { L'imagerie dans la prise } \\
\text { en charge des cancers des voies } \\
\text { aérodigestives supérieures }\end{array}$ & 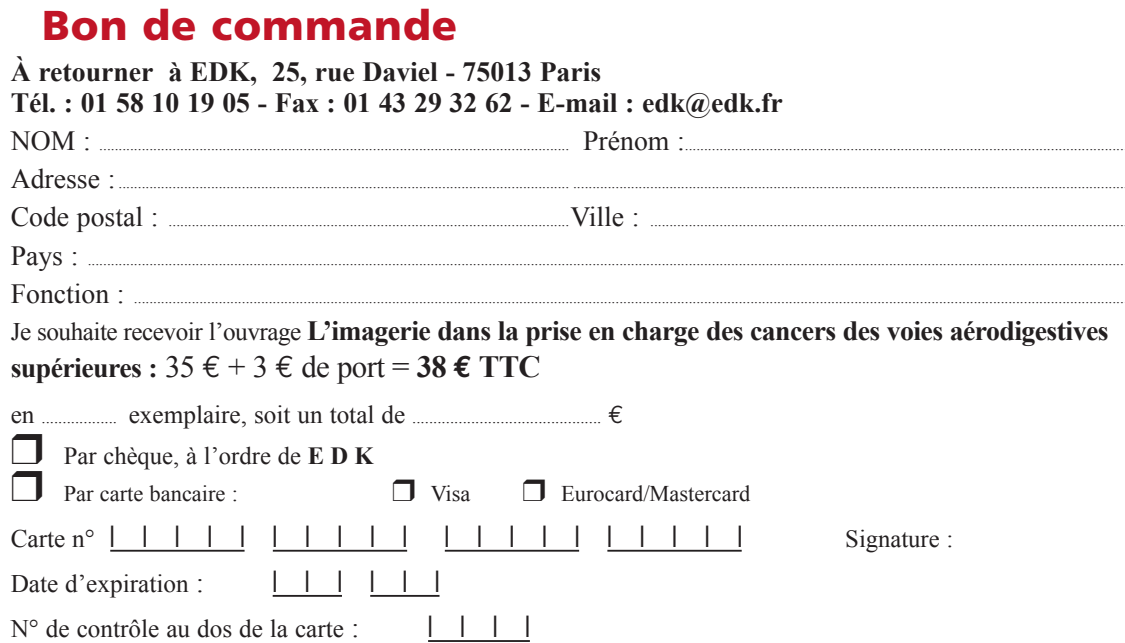 \\
\hline
\end{tabular}

